\title{
Heterodimensional charge-carrier confinement in stacked submonolayer InAs in GaAs
}

\author{
S. Harrison, M. P. Young, P. D. Hodgson, R. J. Young, and M. Hayne* \\ Department of Physics, Lancaster University, Lancaster LA1 4YB, United Kingdom \\ L. Danos \\ Department of Chemistry, Lancaster University, Lancaster LA1 4YB, United Kingdom
}

\author{
A. Schliwa, A. Strittmatter, A. Lenz, H. Eisele, and U. W. Pohl \\ Institut für Festkörperphysik, Technische Universität Berlin, Hardenbergstrasse 36, 10623 Berlin, Germany \\ D. Bimberg \\ Institut für Festkörperphysik, Technische Universität Berlin, Hardenbergstrasse 36, 10623 Berlin, Germany \\ and King Abdulaziz University, Jeddah, Kingdom of Saudi Arabia \\ (Received 7 September 2015; revised manuscript received 11 December 2015; published 2 February 2016)
}

\begin{abstract}
Charge-carrier confinement in nanoscale In-rich agglomerations within a lateral InGaAs quantum well (QW) formed from stacked submonolayers (SMLs) of InAs in GaAs is studied. Low-temperature photoluminescence (PL) and magneto-PL clearly demonstrate strong vertical and weak lateral confinement, yielding two-dimensional (2D) excitons. In contrast, high-temperature (400 K) magneto-PL reveals excited states that fit a Fock-Darwin spectrum, characteristic of a zero-dimensional (0D) system in a magnetic field. This paradox is resolved by concluding that the system is heterodimensional: the light electrons extend over several In-rich agglomerations and see only the lateral InGaAs QW, i.e., are 2D, while the heavier holes are confined within the In-rich agglomerations, i.e., are $0 \mathrm{D}$. This description is supported by single-particle effective-mass and eight-band $k \cdot p$ calculations. We suggest that the heterodimensional nature of nanoscale SML inclusions is fundamental to the ability of respective optoelectronic devices to operate efficiently and at high speed.
\end{abstract}

DOI: 10.1103/PhysRevB.93.085302

\section{INTRODUCTION}

Low-dimensional semiconductor nanostructures, in which quantum confinement can be exploited to deliver desirable optical and electronic properties, have been at the forefront of developments in solid-state physics for more than four decades. Quantum dots (QDs), used as the active medium in lasers, offer the possibility of wavelength tuning and demonstrate ultrafast gain dynamics and better temperature stability, compared to quantum well (QW) lasers [1]. However, the trapping of carriers, for example in wetting layer (WL) states, means that they are not contributing to lasing, which is detrimental to device performance [2,3]. Submonolayer (SML) growth is an alternative to conventional Stranski-Krastanov (SK) growth and results in QD-like In-rich agglomerations without a WL, with areal densities of $10^{12} \mathrm{~cm}^{-2}$, which is much higher than for SK QDs $\left(10^{10}-10^{11} \mathrm{~cm}^{-2}\right)[1,4]$. SML vertical-cavity surface-emitting lasers (VCSELs) have already been shown to operate at over $20 \mathrm{~Gb} \mathrm{~s}^{-1}$ [5], and to fully explain this high-speed performance, one must gain insight into charge carrier confinement within the system.

SML superlattice growth involves the deposition of less than 1 monolayer (ML) of, for example, InAs on GaAs, capping it with a thin (few MLs) spacer layer of GaAs, and repeating the procedure several times to form a SML stack. The technique was first proposed as an alternative method of producing InGaAs QWs [6], but it was subsequently realized that strain could lead to vertical correlations in the growth [7],

*m.hayne@lancaster.ac.uk and the formation of QD structures [8]. InAs has a larger lattice constant than GaAs, so the thin GaAs spacer layer covering each of the partially complete MLs of InAs will locally show tensile strain where it happens to cover an InAs island. This local increase in lattice constant provides a favorable site for the nucleation of the next InAs island [Fig. 1(a)], such that, in the ideal case, vertical alignment of SML InAs islands occurs, forming a system of InGaAs QDs in GaAs [Fig. 1(b)]. Such an alignment has been widely reported for stacked layers of SK self-assembled InAs/GaAs QDs [9,10], and has also been exploited in the fabrication of columnar dots [11] and quantum posts [12]. Electronic coupling in columnar dots, quantum posts, and in stacked layers of QDs $[13,14]$ allows the fabrication of dots with height/base-length aspect ratios of $\sim 1$, or even $>>1$ (quantum wires), with consequent control over the confinement and polarization properties.

Figure 1(c) shows a typical high-resolution cross-sectional scanning-tunneling-microscopy image of a similar SML sample to those studied here. It can be seen that the actual structures are far from the ideal schematic in Fig. 1(b). There is only partial ordering of the InAs, resulting in a disordered InGaAs/GaAs QW, within which are highly nonuniform Inrich agglomerations (highlighted by dotted lines), with a size of very approximately $5 \mathrm{~nm}$. Such a structure immediately raises the question: Is the system two dimensional (QW) or zero dimensional (QD)?

Herein we investigate this question both experimentally and theoretically, and in doing so gain an insight into the complexities of charge-carrier confinement within such a system. Magneto-PL experiments enable us to probe the extent of the exciton and disorder length scales (Secs. III A and III B), 


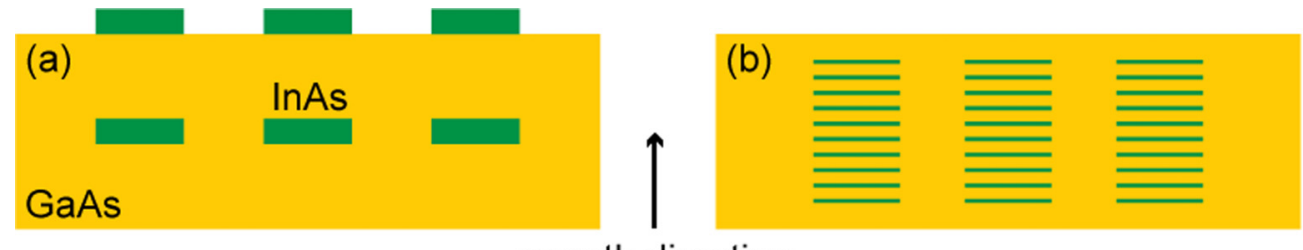

growth direction

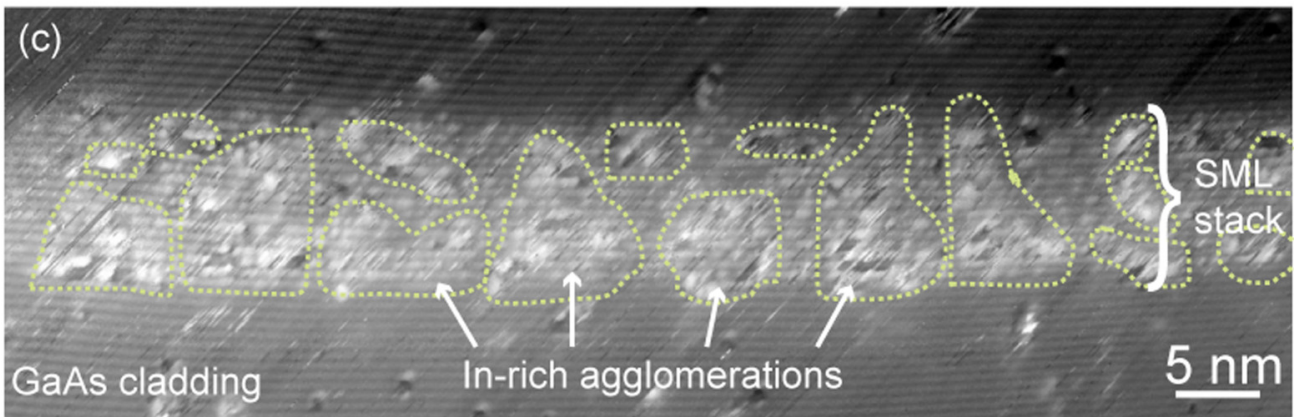

FIG. 1. (a) The successive deposition of 0.5 ML of InAs (green) followed by a few MLs of GaAs (yellow) should, naively, build up a stack of submonolayer InAs islands (b). (c) A cross-sectional scanning-tunneling-microscopy image, taken at $V= \pm 2.9 \mathrm{~V}, I=80 \mathrm{pA}$, showing that, in reality, partial vertical alignment and In segregation leads to highly nonuniform In-rich agglomerations in an InGaAs quantum well. The green dashed lines are guides to the eye.

while time-resolved PL measurements reveal carrier dynamics (Sec. III C). Single-particle effective-mass and eight-band $k \cdot p$ calculations help us verify our experimental measurements (Sec. IV). In Sec. V we discuss the implications of our findings.

\section{SAMPLES AND EXPERIMENTAL DETAILS}

Three SML samples were studied in this investigation, each of which was grown by metalorganic vapor phase epitaxy (MOVPE) at $500{ }^{\circ} \mathrm{C}$ on (001)-oriented GaAs using tertiarybutylarsine, trimethylgallium, and trimethylindium precursors. Tenfold stacks of $0.5 \mathrm{ML}$ of InAs and 1.5, 2.0, or 2.5 MLs of GaAs (samples A, B, and C) were grown in a cyclical procedure applying V/III ratios of 9 and 5, and growth rates of 0.25 and $0.5 \mathrm{ML} \mathrm{s}^{-1}$, respectively, with a 1-s growth interruption prior to and after each InAs deposition. The corresponding stack heights are approximately 11.5, 14.3, and $17.2 \mathrm{~nm}$. The undoped SML structures are surrounded by a 300-nm-thick GaAs matrix which is then sandwiched by $\mathrm{Al}_{x} \mathrm{Ga}_{1-x}$ As barriers to prevent escape of photogenerated carriers. Further details of the growth procedure may be found elsewhere $[15,16]$. As a reference, an undoped 6.5-nm-thick $\mathrm{In}_{0.18} \mathrm{Ga}_{0.82} \mathrm{As} \mathrm{QW}$ on $\mathrm{GaAs}$ was grown at $600{ }^{\circ} \mathrm{C}$ with $\mathrm{V} / \mathrm{III}$ $=10.7$ and a $0.46 \mu \mathrm{m} \mathrm{h}^{-1}$ growth rate. This was similarly sandwiched between $\mathrm{Al}_{x} \mathrm{Ga}_{1-x}$ As barriers.

Magneto-PL experiments were undertaken in a variable temperature insert inside the bore of a superconducting magnet, giving access to fields from 0 to $17 \mathrm{~T}$ at temperatures from 2 to $400 \mathrm{~K}$. Optical access was provided via two multimode optical fibers: one to transmit the excitation light from a 532-nm solid-state laser to the sample, and the second to collect the luminescence. The laser excitation spot on the sample was $\sim 2 \mathrm{~mm}$ and the power was varied from 0.04 to $700 \mathrm{~mW}$, corresponding to laser power densities of $2 \mathrm{~mW} \mathrm{~cm}^{-2}$ to $35 \mathrm{~W} \mathrm{~cm}^{-2}$. The luminescence was analyzed in a $0.3 \mathrm{~m}$ diffraction grating spectrometer fitted with a thermoelectrically cooled InGaAs diode linear array.

Time-resolved PL (TRPL) measurements, all at room temperature, were carried out using a 640-nm laser diode operating at a $40 \mathrm{MHz}$ repetition rate. The TRPL was detected by a Hamamatsu near-infrared photomultiplier tube in the spectral range 950-1400 $\mathrm{nm}$.

\section{EXPERIMENTAL RESULTS}

\section{A. Evidence for a two-dimensional system}

Figure 2 shows low-temperature zero-field PL from the three SML samples and the QW. Immediately striking are the

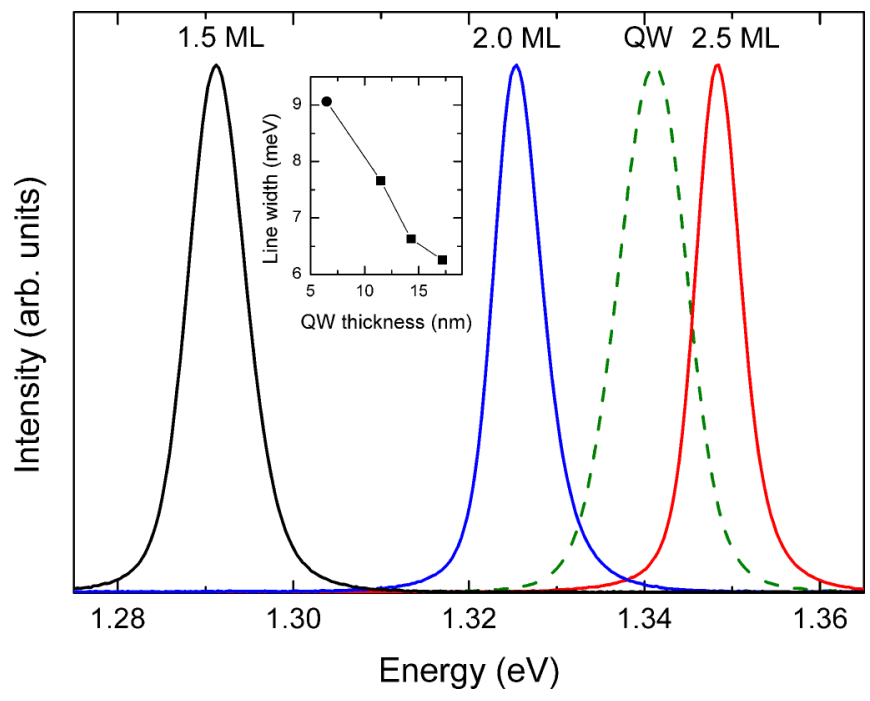

FIG. 2. Low-temperature (2 K) zero-field normalized PL from the three SML (GaAs spacers of 1.5, 2.0, and 2.5 MLs) and the QW samples. The inset shows linewidth dependence on the stack height of the SML samples (or QW thickness, indicated by the circle). 


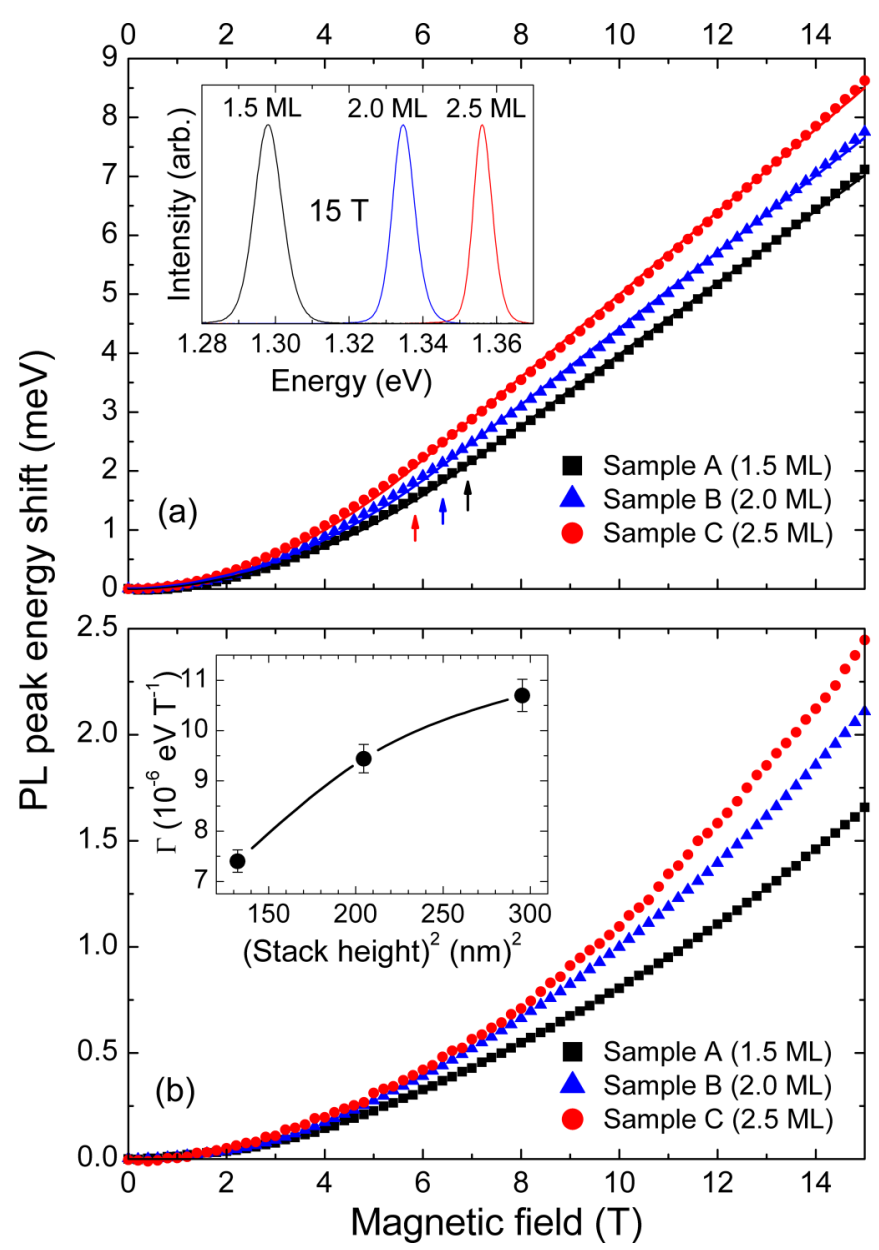

FIG. 3. Low-temperature (2 K) magneto-PL. (a) PL peak energy in Faraday geometry. The crossover from the low- to the high-field regime is indicated by an arrow for each sample. Inset: Individual PL spectra for the three samples at $B=15 \mathrm{~T}$. (b) In Voigt geometry the PL energy is quadratic in $B$ to the highest fields, demonstrating strong vertical confinement. Inset: Dependence of the diamagnetic shift coefficient $\Gamma$ on the square of the stack height in the Voigt geometry.

narrow linewidths (full width at half maximum) of the SML samples $(7.66 \pm 0.01,6.63 \pm 0.01$, and $6.26 \pm 0.01 \mathrm{meV}$ for samples $\mathrm{A}, \mathrm{B}$, and $\mathrm{C}$, respectively), much narrower than is typically the case for self-assembled InAs/GaAs QDs and indeed narrower than that of the QW $(9.06 \pm 0.01 \mathrm{meV})$. This is particularly surprising given the highly nonuniform nature of the In-rich agglomerations in Fig. 1(c), and indicates a QW-like behavior of the SML samples. Furthermore, we see that the PL linewidth decreases with increasing GaAs spacer thickness, i.e., stack height (QW thickness), exactly as might be expected for two-dimensional (2D) confinement [17]: the larger the QW, the smaller the relative size of monolayer fluctuations in QW thickness. This explains why the QW sample, which has the smallest QW thickness, has the broadest PL spectrum (see the inset of Fig. 2).

Figure 3(a) shows the dependence of the PL peak energy (determined by Gaussian fits of the individual spectra) on the magnetic field $B$ with the field applied in the growth direction (Faraday geometry) for the three SML samples. The inset shows the individual PL spectra at $15 \mathrm{~T}$. The data show two regimes: a low-field regime where PL energies demonstrate a $B^{2}$ dependence, and a high-field regime where this dependence is instead linear. We employ the so-called excitonic model [18], in which a crossover between these two regimes occurs at a critical field $B_{\mathrm{c}}$, indicated by an arrow, to fit these dependencies. This model further requires a smooth transition between the regimes at $B_{\mathrm{c}}$, when the magnetic length $(\hbar / e B)^{1 / 2}$ is $1 / \sqrt{2}$ times the exciton Bohr radius $a_{\mathrm{B}}$. Doing so results in the following expressions:

$$
E=E_{0}+\frac{e^{2} a_{\mathrm{B}}^{2} B^{2}}{8 \mu} \quad \text { for } \quad B \leqslant B_{\mathrm{c}}
$$

and

$$
E=E_{0}-\frac{\hbar^{2}}{2 \mu a_{\mathrm{B}}^{2}}+\frac{\hbar e B}{2 \mu} \text { for } B \geqslant B_{\mathrm{c}}
$$

where

$$
B_{\mathrm{c}}=\frac{2 \hbar}{e a_{\mathrm{B}}^{2}} .
$$

$E_{0}$ is the zero-field PL peak energy and $\mu$ is the reduced mass of the exciton. By fitting low- and high-field data simultaneously, one can obtain values for $a_{\mathrm{B}}, \mu$, and the diamagnetic energy shift coefficient $\Gamma=\left(e^{2} a_{\mathrm{B}}^{2}\right) /(8 \mu)$, all of which give an insight into confinement within the system.

Thus we obtain values for lateral Bohr radii (i.e., in the plane of the QW) of $13.8 \pm 0.1,14.3 \pm 0.1$, and $14.9 \pm 0.1 \mathrm{~nm}$, and reduced masses of $(0.095 \pm 0.001) m_{0},(0.090 \pm 0.001) m_{0}$, and $(0.083 \pm 0.001) m_{0}$, where $m_{0}$ is the free electron mass, for the 1.5, 2.0, and 2.5 ML samples, respectively. Hence, the exciton is approximately $30 \mathrm{~nm}$ wide, many times bigger than the In-rich agglomerations, and extending across several of them. The reduced mass decreases for decreasing In content, and we suspect this is the result of an overall reduction in strain in the system when In content is lower, as has been documented before in literature [19-21]. Figure 3(b) shows equivalent data for the field in the QW plane (Voigt geometry), which probes the vertical extent of the exciton (i.e., in the growth direction). The total size of the shift is $\sim 3$ times smaller than for Faraday geometry, and the data show only a quadratic dependence on $B$, i.e., the linear (high- $B$ ) regime is not reached. This unequivocally demonstrates strong confinement $\left[a_{B}<9.4 \mathrm{~nm}\right.$, according to Eq. (3) with maximum $B$ of $\left.15 \mathrm{~T}\right]$ in the growth direction, confirming the $2 \mathrm{D}$ character of the exciton. The inset to Fig. 3(b) shows $\Gamma$ as a function of the square of the stack height. If $\mu$ remains unchanged, and $a_{\mathrm{B}}$ in the growth direction were determined entirely by the stack height, we would get a straight line. Instead a sublinear dependence is observed. However, this is to be expected, since as the stack height increases, the vertical extent of the exciton wave function must saturate at its intrinsic size $\left(a_{B} \sim 8 \mathrm{~nm}\right)$, as determined by the strengths of the Coulomb interaction between electron and hole and their effective masses.

The PL linewidth has briefly been mentioned, and its dependence on spacer-layer thickness attributed to the exciton seeing less of the local fluctuations in QW thickness as spacer layer thickness increases. We now turn our attention to its dependence on $B$, as shown in Fig. 4. As $B$ is increased, the exciton wave function is squeezed and the conventional 


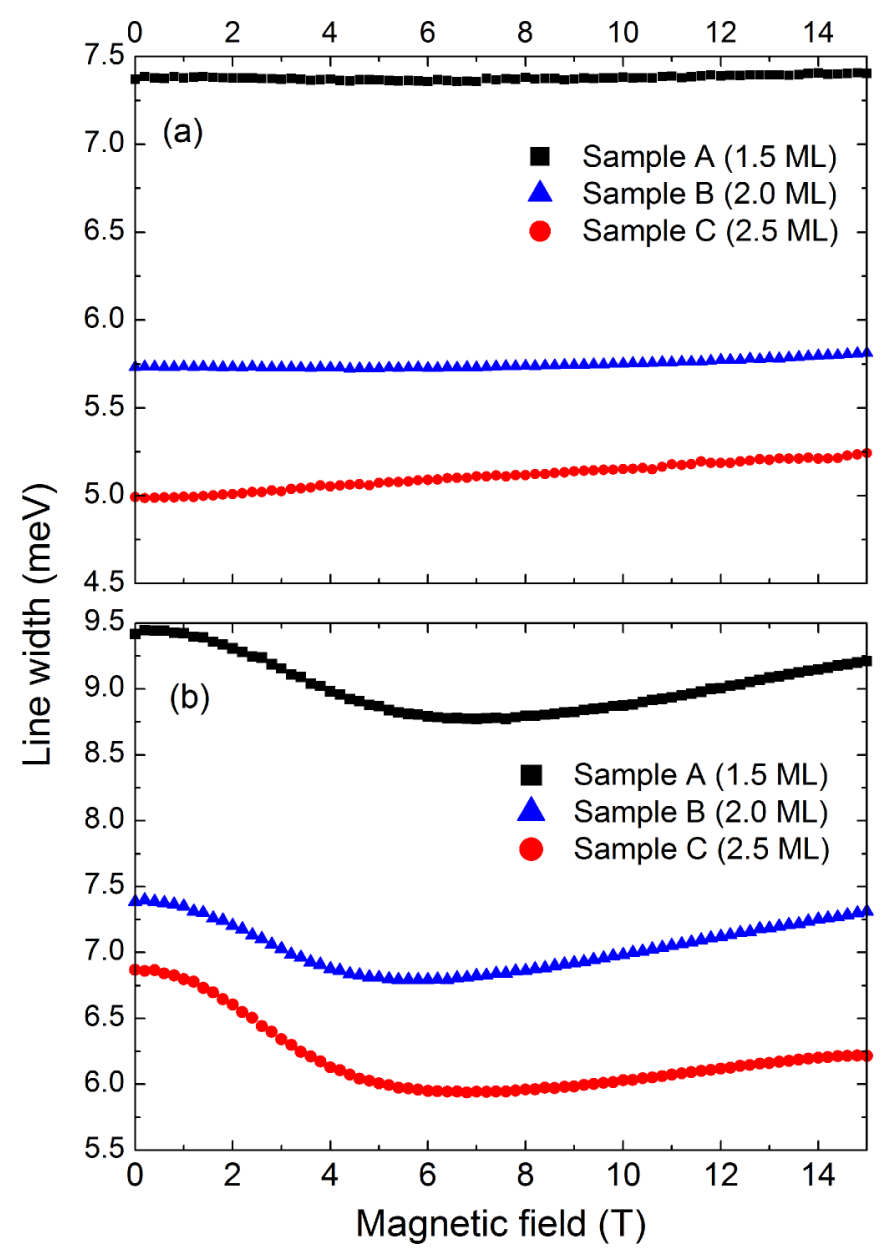

FIG. 4. PL linewidth dependence on $B$. (a) In Voigt geometry, little dependence is seen due to strong vertical confinement (small Bohr radii). (b) In Faraday geometry, the nonmonotonic variation in linewidth with $B$ is the result of two disorder length scales.

argument is that this leads to a reduced disorder averaging and hence an increase in the linewidth [22]. This is based upon the assumption that the correlation length $\chi_{1}$ of the disorder potential is much smaller than $a_{\mathrm{B}}$. Figure 4(a) shows that in Voigt geometry, only sample $\mathrm{C}$, with the greatest spacer layer thickness, exhibits such a dependence and largely, the linewidth is unaffected by the magnetic field. This is consistent with the picture of strong vertical confinement demonstrated by the Voigt-geometry magneto-PL energy data in Fig. 3(b), and can be interpreted as the magnetic field having a marginal effect on the vertical extent of the exciton wave function, and hence, on the disorder probed.

The $B$ dependence of the PL linewidth in Faraday geometry [Fig. 4(b)] is much more interesting, since the lateral extent of the exciton is large. All samples show, up to a field strength of around $6 \mathrm{~T}$, a clear decrease in linewidth, after which the previously explained monotonic increase returns. This behavior can be explained by the presence of a second disorder length scale $\chi_{2}$ [17]. The first correlation length $\chi_{1}$ relates to monolayer fluctuations of the $\mathrm{QW}$, when $\chi_{1}$ is much smaller than $a_{\mathrm{B}}$. The second correlation length $\chi_{2}$ is around the size of $a_{\mathrm{B}}$, and it is reasonable to assume it represents fluctuations in the size of the highly nonuniform In-rich

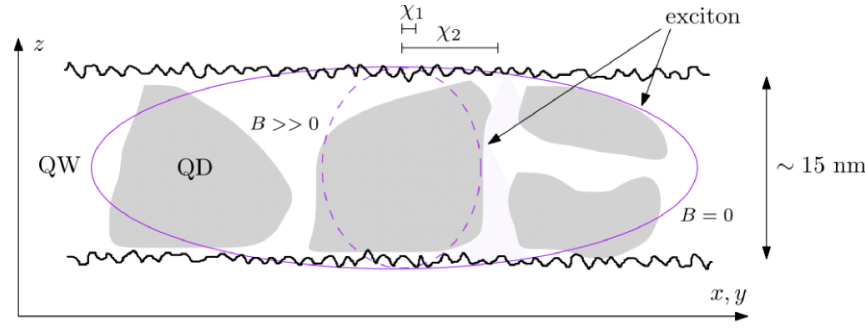

FIG. 5. A schematic representation of the two disorder length scales $\chi_{1}$ and $\chi_{2}$ present in the SML samples. Ellipses illustrate exciton sizes at $B=0$ (solid line) and $B>>0$ (dashed line). Applying a magnetic field squeezes the exciton so it only sees the smaller $\chi_{1}$, which is most likely due to QW thickness. Adapted from Ref. [17].

agglomerations. These larger-scale fluctuations dominate at low $B$, until the wave function is squeezed to an extent that it only sees the smaller-scale fluctuations described by $\chi_{1}$, and hence we see linewidth dependencies with low- and high-field regimes. This fits with the picture painted above, of excitons being confined within a lateral QW, with wave functions extending across several In-rich agglomerations, and is represented schematically in Fig. 5. If it was the case that excitons were zero dimensional (0D), their wave function would not see the larger scale fluctuations described by $\chi_{2}$.

The reader may note that the zero-field PL linewidths for the individual samples in Faraday and Voigt geometry are different. An explanation for this lies within systematic errors from our measurement setup. In Faraday geometry, excitation light is shone straight from an optical fiber to the sample, while in Voigt geometry, the light is first reflected $90^{\circ}$ by a mirror. In this geometry, the light hits many different areas of the sample, systematically increasing linewidths.

So far we have unequivocally demonstrated the 2D nature of the exciton: zero-field PL linewidths are QW-like and depend on stack height, lateral Bohr radii extend over several In-rich agglomerations, vertical confinement is strong and governed by the stack height, and linewidth dependencies on magnetic field show evidence for two disorder length scales.

\section{B. Evidence for a zero-dimensional system}

Figure 6(b) shows a PL spectrum for sample $\mathrm{C}$ taken at $17 \mathrm{~T}$ and $400 \mathrm{~K}$. In contrast to the low-temperature SML data, a number of excited-state peaks can be resolved at high temperature. The field dependence of the main PL peak and the two most prominent excited-state peaks is plotted in Fig. 6(a) (Faraday geometry). The peak positions can be fitted with a Fock-Darwin spectrum (solid lines), revealing behavior characteristic of a 0D system. The Fock-Darwin model describes the energy levels of a particle in a two-dimensional harmonic potential of energy $\hbar \omega_{0}$ in a perpendicularly applied magnetic field, but given the atom-like properties QDs possess, it proves a good (single-particle) approximation for a QD in a magnetic field $[18,23]$. For Landau-level number $n(n=0,1,2, \ldots)$ and orbital angular momentum $l(l=0, \pm 1, \pm 2, \ldots)$, the $(n, l)$ th energy level is given by

$$
E_{n, l}=E_{C}+(2 n+|l|+1) \hbar \sqrt{\omega_{0}^{2}+\frac{\omega_{\mathrm{c}}^{2}}{4}}-\frac{1}{2} l \hbar \omega_{\mathrm{c}},
$$




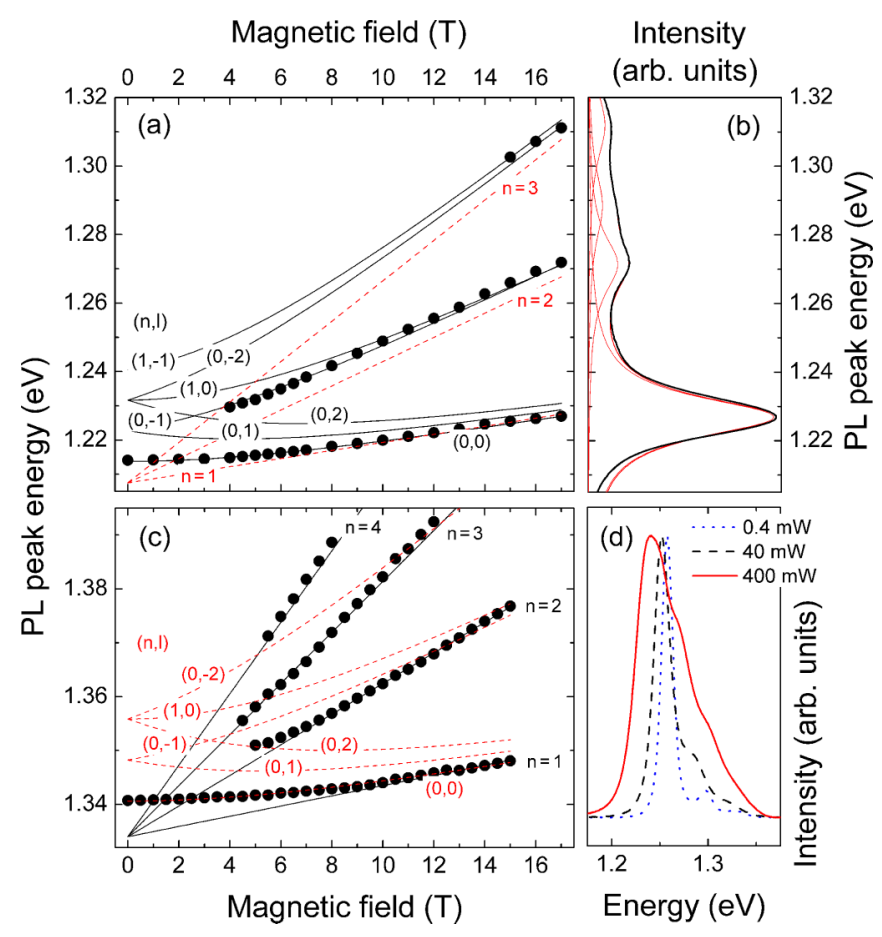

FIG. 6. (a) High-temperature ( $400 \mathrm{~K}$ ) magnetic-field dependence of the PL peaks (Faraday geometry) for sample C. The solid lines are fits to a Fock-Darwin spectrum with Landau-level index $n$ and orbital angular momentum $l$. The confinement energy $\hbar \omega_{0}$ derived from the fits is found to be $\sim 9 \mathrm{meV}$ for all samples. The (red) dashed lines show the best attempt at a Landau-Level fit. (b) 400-K 17-T spectrum (thick line) with fitted peaks (thin lines), for sample C. (c) Low-temperature ( $2 \mathrm{~K}$ ) magnetic-field dependence of the PL peaks (Faraday geometry) for the QW sample. The solid lines are Landau level fits while the (red) dashed lines are a best-attempt Fock-Darwin fit. (d) 15-T 300-K PL spectra for the QW sample, showing a strong dependence on excitation laser power.

where $E_{C}$ is the confinement energy in the direction of the magnetic field, $\omega_{\mathrm{c}}=|e B| / \mathrm{m}^{*}$ is the cyclotron frequency due to this field, and $m^{*}$ is the effective mass of the particle. The data fitted in Fig. 6(a) yield an exciton effective mass of $(0.051 \pm 0.001) m_{0}$, where $m_{0}$ is the free electron mass. Similar behavior was observed for all three samples at similar temperatures, i.e., all three SML samples show clear evidence for $0 \mathrm{D}$ properties at elevated temperatures, with a confinement energy $\hbar \omega_{0}$ of $\sim 9 \mathrm{meV}$.

\section{Comparison with quantum well sample}

Further confirmation of the unusual behavior of the SML samples is obtained by measuring the magnetic field dependence of excited states in the QW, and comparing it with the SML samples. However, while the SML samples show little dependence on laser power, the line shape and width of the QW PL spectra are greatly affected: higher powers see the increased prominence of excited-state peaks, and at high temperature this leads to a broad PL spectrum that is difficult to resolve [Fig. 6(d)]. Optical pumping by the laser clearly has a greater effect in the QW, and this is attributed to a shorter carrier lifetime in the SML samples, as verified

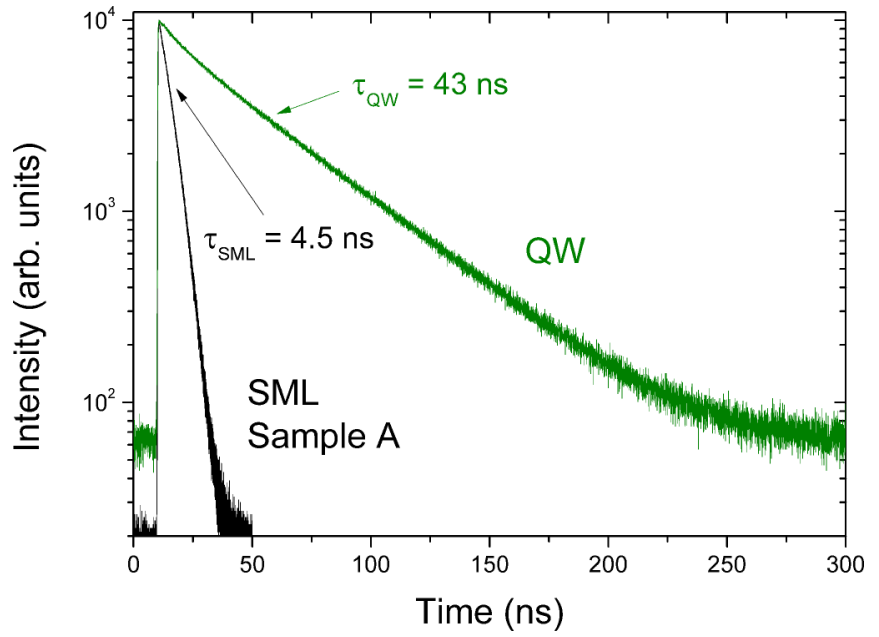

FIG. 7. Room-temperature time-resolved PL measured at the PL peak wavelength for sample A (black, measured at $1029 \mathrm{~nm}$ ) and the QW sample (green, measured at $986 \mathrm{~nm}$ ). Intensity-weighted average carrier lifetimes $\tau_{\mathrm{av}}$ are indicated by the arrows, showing the SML carrier lifetime is approximately an order of magnitude shorter.

by room-temperature TRPL measurements (Fig. 7). The SML samples show an approximately order-of-magnitude shorter carrier lifetime than the QW (similar for all SML samples). The SML time-resolved data also show a slightly convex shape, indicating that the rate of decay is time dependent. This highlights the complex dynamics of carrier distribution after the initial optical pumping by the laser in the SML samples, and this effect is not seen for the QW. For these reasons we compare the field dependence of the excited states in the SML samples at $300 \mathrm{~K}$ with the excited states of the QW sample at $2 \mathrm{~K}$.

In contrast to the SML samples, the field dependence of excited-state PL peak energies for the QW sample could not be fitted by a Fock-Darwin spectrum, and instead, lowtemperature PL peak energies in the high-field regime follow a Landau level fit [Fig. 6(c)], for which energy levels are described by

$$
E=E_{C}+\left(n+\frac{1}{2}\right) \hbar \omega_{\mathrm{c}},
$$

yielding an exciton effective mass of $(0.061 \pm 0.001) m_{0}$.

Finally, we turn our attention to the temperature dependence of the PL data, shown in Fig. 8. At high powers, both the QW and SML samples show a strong Varshni-like temperature dependence and fitting reveals Varshni [24] parameters of $\alpha=0.49 \pm 0.01 \mathrm{meV} \mathrm{K}^{-1}$ and $\beta=180 \pm 10 \mathrm{~K}$, between those for InAs $\left(\alpha=0.276 \mathrm{meV} \mathrm{K}^{-1}, \beta=93 \mathrm{~K}\right)$ and $\mathrm{GaAs}$ $\left(\alpha=0.541 \mathrm{meV} \mathrm{K}^{-1}, \beta=204 \mathrm{~K}\right)$ [25]. At lower powers an S-shaped dependence develops (Fig. 8), commonly attributed to potential fluctuations in the InGaAs QW layer [26-28]. Though there is little difference between the QW and SML temperature dependencies [29], it can be seen that the S-shaped curve is slightly more pronounced for the QW. This effect is small compared to, for example, SML samples in which $\mathrm{Sb}$ is incorporated [30], so these results are consistent with our findings that the low-temperature excitonic properties of the SML samples are largely unaffected by the In-rich agglomerations. Indeed, according to this data the effect of 


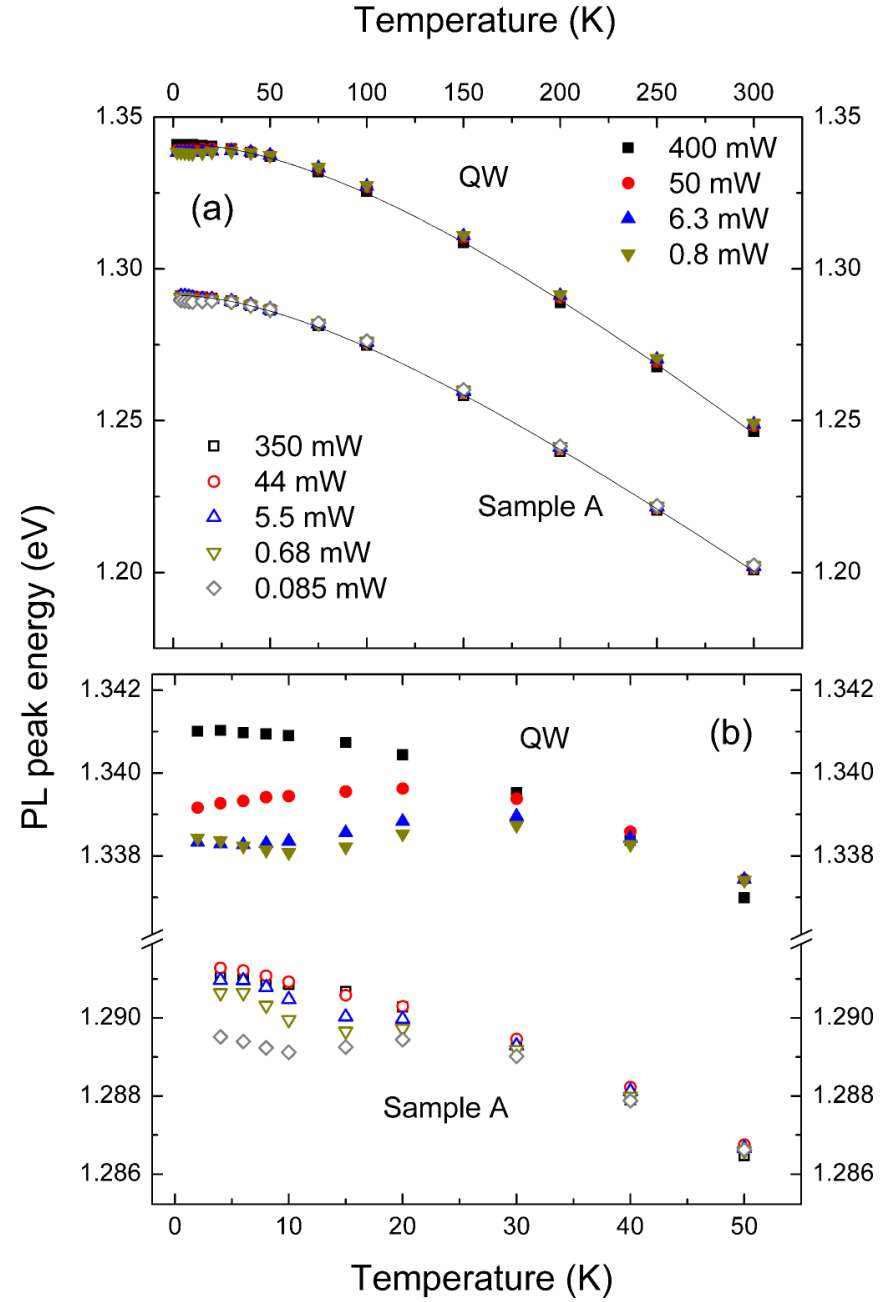

FIG. 8. Temperature dependence of PL peak energy. (a) For sample A and the QW at various laser powers. The solid lines are Varshni [24] fits of the highest laser powers, 400 and $350 \mathrm{~mW}$ for the QW and SML sample, respectively. (b) The same data as (a) but focused on $0-50 \mathrm{~K}$ to highlight the S-shaped dependence on temperature at lower powers.

compositional fluctuations on excitonic properties is even less for the SML samples than for the QW sample.

\section{Heterodimensionality}

Summarizing the experimental results, we have presented strong evidence that the excitons in the SML samples are 2D (low-temperature PL) and, in apparent contradiction, we have also observed 0D states (high-temperature PL). We resolve this paradox by proposing that stacked layers of SML InAs islands in GaAs form a heterodimensional system. Heavy holes are confined to In-rich agglomerations with confinement energies of $\sim 9 \mathrm{meV}$ (holes see a 0D system), while the electrons are too light to be confined in these dotlike regions and instead see only the lateral InGaAs QW (a 2D system). Since the electrons are the lighter, much more extended charge carriers, they define the optical properties of the exciton, such that (low-temperature) optical experiments (linewidth, exciton extent, etc.) reveal the $2 \mathrm{D}$ character of the system, while in high-temperature experiments there is population of excited confined states of the holes, allowing observation of their OD nature.

With this postulation, the Fock-Darwin fit presented in Fig. 6(a) can be treated slightly differently. When Eq. (4) is fitted to PL data, $\hbar \omega_{0}$ is a sum of electron and hole confining energies, $\hbar \omega_{0}=\hbar\left(\omega_{0}^{\mathrm{e}}+\omega_{0}^{\mathrm{h}}\right)$, and the electron and hole effective masses $m_{\mathrm{e}}^{*}$ and $m_{\mathrm{h}}^{*}$ are contained within the reduced exciton mass $\mu=1 /\left(1 / m_{\mathrm{e}}^{*}+1 / m_{\mathrm{h}}^{*}\right)$ [23]. For a heterodimensional system, $\hbar \omega_{0}^{\mathrm{e}}=0$ and so the $\sim 9 \mathrm{meV}$ confinement energy seen in our samples must come solely from the holes $\left(\hbar \omega_{0}^{\mathrm{h}}=9 \mathrm{meV}\right)$. Fitting our data with this modified Fock-Darwin description verifies that a heterodimensional system can produce a Fock-Darwin spectrum, reproducing the curves in Fig. 6 [31].

\section{MODELING THE SYSTEM}

Having presented our experimental data we now go on to discuss two different sets of calculations, both of which fully support the concept of heterodimensionality proposed for this system. First, single-particle effective-mass calculations without strain solve the Schrödinger equation for each band separately, providing qualitative support for heterodimensionality and gaining an insight into the parameter space in terms of (differential) In composition for which heterodimensionality may occur. The samples are modeled as a spherical 5-nmdiameter $\mathrm{In}_{x} \mathrm{Ga}_{1-x}$ As QD in a 13-nm-high $\mathrm{In}_{y} \mathrm{Ga}_{1-y} \mathrm{As}$ QW [Fig. 9(a)], with a conduction-band:valence-band offset of 60:40 and band parameters from Ref. [25]. Figure 9(b) shows a representative calculation for $x=0.50$ and $y=0.15$. As predicted, the electron's ground state lies in the $\mathrm{QW}$, while the hole is confined in the QD. In order to explore this further, and as we do not have values for the In composition of the

(a)

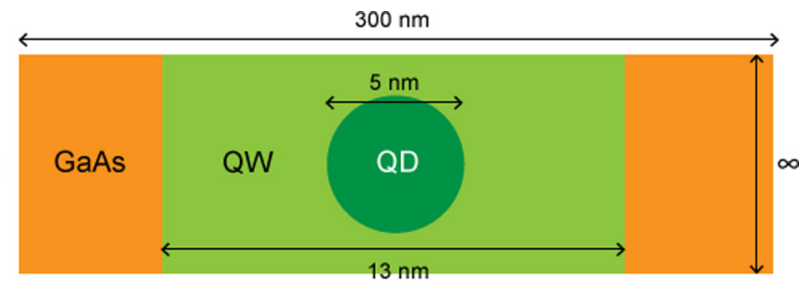

(b)
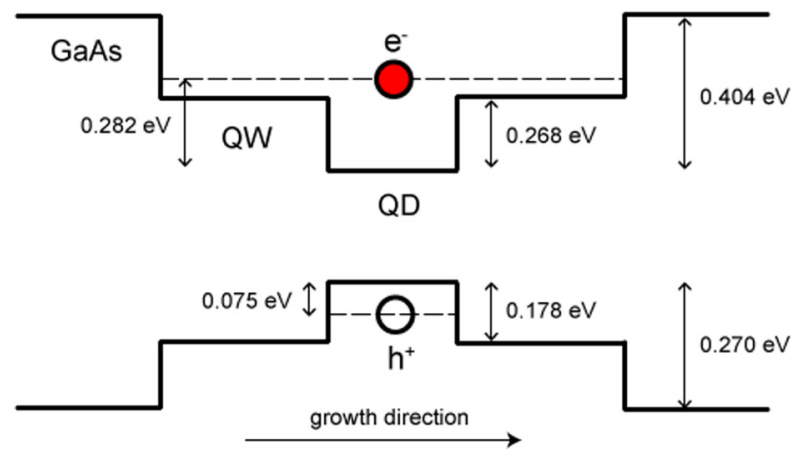

FIG. 9. Effective mass calculations. (a) Schematic representation of the modeled system as a 5-nm, spherical $\operatorname{In}_{x} \mathrm{Ga}_{1-x} \mathrm{As}$ QD in a 13-nm-high $\operatorname{In}_{y} \mathrm{Ga}_{1-y} \mathrm{As}$ QW. (b) Calculated electron and hole confined states for $x=0.50$ and $y=0.15$. The hole is confined by the $\mathrm{QD}$, but the lowest confined electron state is bound by the QW. 


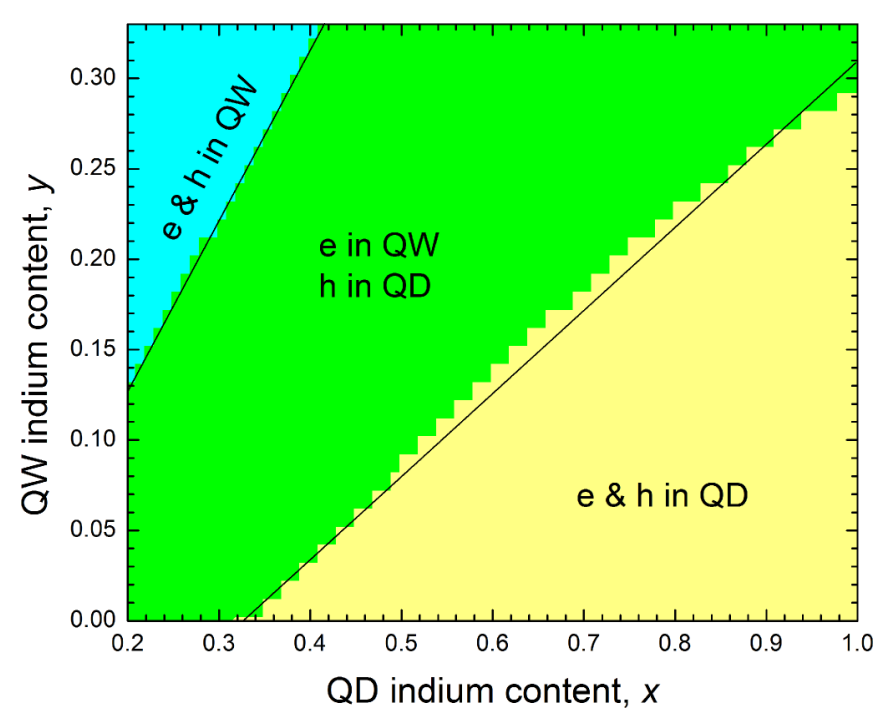

FIG. 10. Effective-mass calculation of electron e and hole $h$ confinement for 5-nm, spherical $\operatorname{In}_{x} \mathrm{Ga}_{1-x}$ As QDs in a 13-nm $\mathrm{In}_{y} \mathrm{Ga}_{1-y} \mathrm{As} \mathrm{QW}$, with varying In content. The shaded regions are defined by the calculation, while the solid lines are guides to the eye indicating the approximate positions of the phase boundaries. The heterodimensional phase (green, 2D electrons and 0D holes) occupies the most likely regions of the parameter space.

samples, $x$ and $y$ were then varied over a large parameter space of In composition $(0.2<x<1$ and $0<y<0.33)$ and a confinement "phase diagram" constructed (Fig. 10). The phase diagram has three regions, one in which both electron and hole are 2D, one in which they are both 0D, and a heterodimensional region where electrons see a $\mathrm{QW}$ and holes see a QD. In order to confine the electrons as well as the holes in the In-rich agglomerations (QDs), very strong clustering of the In is required. For 5-nm spherical In-rich agglomerations it is necessary for $x$ to be at least $2.2 y+0.33$. Similarly, for the holes to see a QW the In composition must be very uniformly distributed, with almost no clustering at all $(x<1.1 y+0.06)$. The implication is that heterodimensional confinement is almost inevitable in this system, and this is consistent with its observation in all three SML samples.

It is important to note that Fig. 10 characterizes the electron and hole ground states, and is thus strictly valid at $0 \mathrm{~K}$. At higher temperatures, e.g., $400 \mathrm{~K}$, thermal energy $k_{B} T$ is $\sim 35 \mathrm{meV}$, allowing excited states to be occupied. The effect of this is an increase in the size of the blue region in Fig. 10, i.e., 2D confinement of electrons and holes becomes more likely. Conversely, for systems with a large QD:QW In content ratio, higher temperatures are likely to push electrons out of the QD sooner as this ratio decreases, making heterodimensionality more likely (i.e., decreasing the size of the yellow region in Fig. 10). However, the overall consequence of this effect is small and, importantly, the heterodimensional phase of Fig. 10 still occupies the most probable parameter space. That this is the case is demonstrated by the experimental observation of OD states in all SML samples at $400 \mathrm{~K}$.

For further insight, in particular to judge the effects of lateral coupling and the Coulomb interaction between electrons and holes, we performed a much more sophisticated
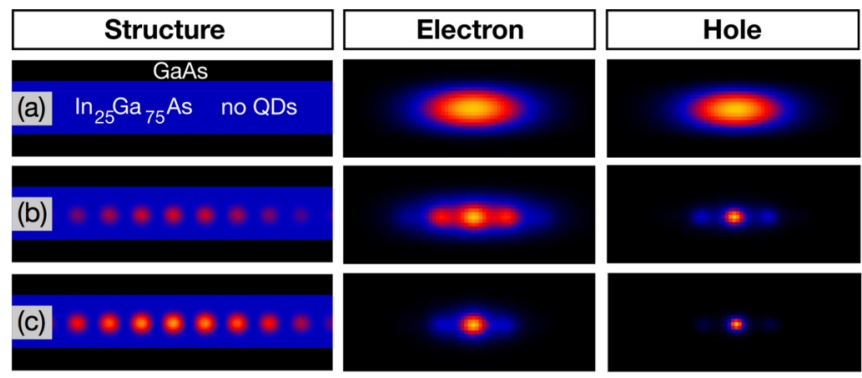

FIG. 11. Color contour plots of electron and hole wave functions in a 14-nm $\operatorname{In}_{0.25} \mathrm{Ga}_{0.75} \mathrm{As}$ QW with (a) no QDs, (b) an array of QDs with $x \leqslant 0.5$, and (c) an array of QDs with $x \leqslant 0.75$. The In composition is maximum in the center of each dot and follows a Gaussian profile across the array. The left-hand panel shows the morphology of the structure, while the middle and right-hand panels show the electron and hole wave functions, respectively.

calculation of a periodic $2 \mathrm{D}$ array of $\sim 5-\mathrm{nm}$ diameter dots in an $\operatorname{In}_{0.25} \mathrm{Ga}_{0.75} \mathrm{As}$ QW using eight-band $k \cdot p$ theory [32] in conjunction with a self-consistent Hartree approach. In this idealized 3D morphology, strain, piezoelectricity, and Coulomb interaction are fully taken into account. Figure 11 shows the results of such a calculation. In the plane of the well the In-rich agglomerations are modeled by In content varying as $\cos ^{2}$ functions in the two orthogonal directions in the plane of the sample, superposed by a broad Gaussian variation in In content over all the dots, such that peak In content in the center dot is 0.5 and 0.75 in Figs. 11(b) and 11(c), respectively. The variation in In content in different dots mimics their morphological diversity while ensuring that the exciton is centered on the QD in the middle. The results are consistent with the effective-mass calculations and the experimental data. The electron is delocalized over several QDs, but eventually gets localized at high In content $(x \sim 0.6)$. Localization of the holes is delayed by lateral coupling, but the hole is consistently much more localized than the electron. The Coulomb interaction enhances electron localization, but this is insufficient to eliminate the heterodimensional behavior. Since the electrons are more extended, the Coulomb interaction has less effect on the holes, which are already localized by the QDs.

\section{DISCUSSION}

Having unequivocally demonstrated, both experimentally and theoretically, the coexistence of $0 \mathrm{D}$ and 2D confinement in stacked SML InAs in GaAs, we go on to discuss some of its implications.

We contend that heterodimensionality is fundamental to the high-speed ( $>20 \mathrm{~Gb} \mathrm{~s}^{-1}$ ) operation of SML VCSELs that has already been demonstrated [5]. In conventional QD lasers, the localization of carriers in confined states that do not contribute to lasing is detrimental to laser operation. Here the 2D electrons couple to several In-rich agglomerations simultaneously and are therefore easily available to agglomerations that are lasing. The confinement of holes in QDs still permits some advantages of the reduced density of states to be exploited, while the relatively shallow nature of the QD confining potential ( $Q W$ barriers) and the small size of the In-rich agglomerations mean 
that large numbers of excess holes cannot build up in nonlasing states (where undesirable nonradiative recombination can occur). Furthermore, the absence of a WL, which can trap carriers and slow down injection into lasing states, reduces the number of these nonlasing states and leads to a high modal gain [33].

Coupling of excited electron QD states has already been used in the InAs/InP system to improve laser performance, but this involved a somewhat more complex stacking procedure to encourage the formation of a lateral superlattice [34], and would have an insignificant effect on the hole distribution. Similarly, it has also been demonstrated that the inclusion of an InGaAs QW injector layer close to InAs QDs allows rapid tunneling of electrons into the lasing QD states, improving the modulation bandwidth of near-infrared QD lasers from $\sim 7$ to $\sim 22 \mathrm{GHz}$ [3]. The stacked SML InAs in GaAs systems studied here offer the advantage that they are simple and easier to fabricate. A high QD density (much higher than conventionally grown SK QDs [1,4]) is characteristic of their growth, meaning not only are the $2 \mathrm{D}$ electrons available to a number of lasing QD states, but the number of these states is large, improving the gain. In semiconductor optical amplifiers based on stacked SML InAs in GaAs, a fast gain recovery was recently demonstrated [35].

Thinking more broadly, strong localization of carriers in compositional fluctuations in QWs is also prevalent in nitride systems, both in InGaN/GaN QWs [36] and in dilute nitrides [37]. Indeed, it is widely argued that carrier localization in InGaN/GaN QW devices is crucial to their operation, as it inhibits nonradiative recombination at dislocations [38]. The heterodimensional concept which we describe here could provide new insight into the physics of such systems, with consequent improvements in device properties for a broad range of applications.
Equally as important is the demonstration that the OD properties of the system are robust up to high temperatures (400 K). Indeed, it was only working at these high temperatures that allowed the population of excited confined hole states, rendering them visible. The potential for efficient hightemperature operation is one of the key advantages of QD lasers [39], and the striking observation of the robust nature of the $0 \mathrm{D}$ density of states at $400 \mathrm{~K}$ is a clear validation of this vision.

\section{CONCLUSIONS}

We have shown that stacked SML InAs in GaAs forms a heterodimensional system, in which holes are confined to In-rich QD-like agglomerations while electrons see only a uniform lateral InGaAs QW. The exitonic characteristics of the system are governed primarily by the light, extended, 2D electrons, while high temperatures and magnetic fields enable us to probe $0 \mathrm{D}$ hole states. We suggest this confinement is fundamental to high-speed ( $>20 \mathrm{~Gb} \mathrm{~s}^{-1}$ ) operation of SML VCSELs [5]. Furthermore, the heterodimensional concept demonstrated here could provide new insight into other technologically important systems in which composition-driven carrier localization plays a role.

\section{ACKNOWLEDGMENTS}

We would like to acknowledge David Quandt from the Institut für Festkörperphysik, Technische Universität Berlin, for general MOVPE support. This work was supported by the Engineering and Physical Sciences Research Council and Deutsche Forschungsgemeinschaft within CRC 787. The data in the figures for this manuscript are openly available from Lancaster University data archive at http://dx.doi.org/10.17635/lancaster/researchdata/26.
[1] D. Bimberg, N. Kirstaedter, N. N. Ledentsov, Z. I. Alferov, P. S. Kop'ev, and V. M. Ustinov, J. Sel. Top. Quantum Electron. 3, 196 (1997)

[2] M. Kuntz, M. L. G. Fiol, D. Bimberg, M. G. Thompson, K. T. Tan, C. Marinelli, A. Wonfor, R. Sellin, R. V. Penty, I. H. White, V. M. Ustinov, A. E. Zhukov, Y. M. Shernyakov, A. R. Kovsh, N. N. Ledentsov, C. Schubert, and V. Marembert, New J. Phys. 6, 181 (2004).

[3] S. Fathpour, Z. Mi, and P. Bhattacharya, J. Phys. D: Appl. Phys. 38, 2103 (2005).

[4] S. Gaan, G. He, R. M. Feenstra, J. Walker, and E. Towe, J. Appl. Phys. 108, 114315 (2010).

[5] F. Hopfer, G. Fio, M. Kuntz, V. A. Shchukin, V. A. Haisler, T. Warming, E. Stock, S. S. Mikhrin, I. L. Krestnikov, D. A. Livshits, A. R. Kovsh, C. Bornholdt, A. Lenz, H. Eisele, M. Dahne, N. N. Ledentsov, and D. Bimberg, IEEE J. Sel. Top. Quantum Electron. 13, 1302 (2007).

[6] A. Y. Egorov, A. E. Zhukov, P. S. Kop'ev, N. N. Ledentsov, M. V. Maksimov, and V. M. Ustinov, Semiconductors 28, 363 (1994).

[7] V. A. Shchukin, D. Bimberg, V. G. Malyshkin, and N. N. Ledentsov, Phys. Rev. B 57, 12262 (1998).
[8] A. E. Zhukov, A. R. Kovsh, S. S. Mikhrin, N. A. Maleev, V. M. Ustinov, D. A. Livshits, I. S. Tarasov, D. A. Bedarev, M. V. Maximov, A. F. Tsatsul'nikov, I. P. Soshnikov, P. S. Kop'ev, Z. I. Alferov, N. N. Ledentsov, and D. Bimberg, Electron. Lett. 35, 1845 (1999).

[9] Q. Xie, A. Madhukar, P. Chen, and N. P. Kobayashi, Phys. Rev. Lett. 75, 2542 (1995).

[10] N. N. Ledentsov, V. A. Shchukin, M. Grundmann, N. Kirstaedter, J. Böhrer, O. Schmidt, D. Bimberg, V. M. Ustinov, A. Y. Egorov, A. E. Zhukov, P. S. Kop'ev, S. V. Zaitsev, N. Y. Gordeev, Z. I. Alferov, A. I. Borovkov, A. O. Kosogov, S. S. Ruvimov, P. Werner, U. Gösele, and J. Heydenreich, Phys. Rev. B 54, 8743 (1996).

[11] P. Ridha, L. H. Li, M. Mexis, P. M. Smowton, J. Andrzejewski, G. Sęk, J. Misiewicz, E. P. O'Reilly, G. Patriarche, and A. Fiore, IEEE J. Quantum Electron. 46, 197 (2010).

[12] D. Alonso-Alvarez, J. M. Ripalda, B. Alen, J. M. Llorens, A. Rivera, and F. Briones, Adv. Mater. 23, 5256 (2011).

[13] M. Colocci, A. Vinattieri, L. Lippi, F. Bogani, M. Rosa-Clot, S. Taddei, A. Bosacchi, S. Franchi, and P. Frigeri, Appl. Phys. Lett. 74, 564 (1999). 
[14] J. Maes, M. Hayne, M. Henini, F. Pulizzi, A. Patané, L. Eaves, and V. V. Moshchalkovi, Physica B 346-347, 428 (2004).

[15] A. Lenz, H. Eisele, J. Becker, L. Ivanova, E. Lenz, F. Luckert, K. Pötschke, A. Strittmatter, U. W. Pohl, D. Bimberg, and M. Dähne, Appl. Phys. Express 3, 105602 (2010).

[16] A. Lenz, H. Eisele, J. Becker, J.-H. Schulze, T. D. Germann, F. Luckert, K. Pötschke, E. Lenz, L. Ivanova, A. Strittmatter, D. Bimberg, U. W. Pohl, and M. Dähne, J. Vac. Sci. Technol. 29, 04D104 (2011).

[17] B. Bansal, M. Hayne, B. M. Arora, and V. V. Moshchalkov, Appl. Phys. Lett. 91, 251108 (2007).

[18] M. Hayne and B. Bansal, Luminescence 27, 179 (2012).

[19] S. Khattak, Ph.D. thesis, Lancaster University, 2015.

[20] M. Yahyaoui, K. Sellami, S. B. Radhia, K. Boujdaria, M. Chamarro, B. Eble, C. Testelin, and A. Lemaître, Semicond. Sci. Technol. 29, 075013 (2014).

[21] C. Pryor, Phys. Rev. B 57, 7190 (1998).

[22] J. B. B. de Oliveira, E. A. Meneses, and E. C. F. da Silva, Phys. Rev. B 60, 1519 (1999).

[23] A. Babinski, M. Potemski, S. Raymond, J. Lapointe, and Z. R. Wasilewski, Phys. Status Solidi 3, 3748 (2006).

[24] Y. P. Varshni, Physica 34, 149 (1967).

[25] I. Vurgaftman, J. R. Meyer, and L. R. Ram-Mohan, J. Appl. Phys. 89, 5815 (2001).

[26] R. Heitz, I. Mukhametzhanov, A. Mudhukar, A. Hoffmann, and D. Bimberg, J. Electron. Mater. 28, 520 (1999).

[27] Q. Li, S. J. Xu, M. H. Xie, and S. Y. Tong, J. Phys.: Condens. Matter 17, 4853 (2005).

[28] V. K. Dixit, S. Porwal, S. D. Singh, T. K. Sharma, S. Ghosh, and S. M. Oak, J. Phys. D 47, 65103 (2014).

[29] The QW was designed to have a similar In content to the SML samples and so one would expect a similar temperature dependence.
[30] D. Quandt, J.-H. Schulze, A. Schliwa, Z. Diemer, C. Prohl, A. Lenz, H. Eisele, A. Strittmatter, U. W. Pohl, M. Gschrey, S. Rodt, S. Reitzenstein, D. Bimberg, M. Lehmann, and M. Weyland, Phys. Rev. B 91, 235418 (2015).

[31] Actual values for electron and hole effective masses are not reported since the large number of parameters and lack of knowledge of actual In content makes uncertainties in the value from the fit large.

[32] O. Stier, M. Grundmann, and D. Bimberg, Phys. Rev. B 59, 5688 (1999).

[33] Z. Xu, D. Birkedal, M. Juhl, and J. M. Hvam, Appl. Phys. Lett. 85, 3259 (2004).

[34] C. Cornet, M. Hayne, P. Caroff, C. Levallois, L. Joulaud, E. Homeyer, C. Paranthoen, J. Even, C. Labbé, H. Folliot, V. V. Moshchalkov, and S. Loualiche, Phys. Rev. B 74, 245315 (2006).

[35] B. Herzog, N. Owschimikow, J.-H. Schulze, R. Rosales, Y. Kaptan, M. Kolarczik, T. Switaiski, A. Strittmatter, D. Bimberg, U. W. Pohl, and U. Woggon, Appl. Phys. Lett. 107, 201102 (2015).

[36] D. Watson-Parris, M. J. Godfrey, P. Dawson, R. A. Oliver, M. J. Galtrey, M. J. Kappers, and C. J. Humphreys, Phys. Rev. B 83, 115321 (2011)

[37] T. Nuytten, M. Hayne, B. Bansal, H. Y. Liu, M. Hopkinson, and V. V. Moshchalkov, Phys. Rev. B 84, 045302 (2011).

[38] S. Hammersley, D. Watson-Parris, P. Dawson, M. J. Godfrey, T. J. Badcock, M. J. Kappers, C. McAleese, R. A. Oliver, and C. J. Humphreys, J. Appl. Phys. 111, 83512 (2012).

[39] A. E. Zhukov, A. Y. Egorov, A. R. Kovsh, V. Maksmiov, A. F. Tsatsul'nikov, N. N. Ledentsov, N. Y. Gordeev, S. V. Ză̆tsev, P. S. Kop'ev, and Z. I. Alferov, Semiconductors 31, 84 (1997). 